Edited by:

Angelique Bordey, Yale University School of Medicine, USA

Reviewed by:

Tatsuhiro Hisatsune, The University of

Tokyo, Japan

${ }^{\star}$ Correspondence:

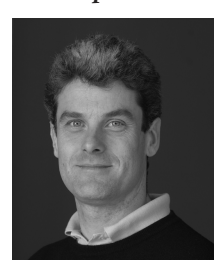

Gerd Kempermann, born in Cologne, Germany, in 1965 is Professor at the CRTD, Center of Regenerative Therapies in Dresden, and speaker of the Dresden partner site of the DZNE, German Center for Neurodegenerative Diseases. His research interest are the activitydependent regulation of adult hippocampal neurogenesis and its molecular control as well as the functional relevance of adult-generated neurons. He is the author of the monograph "Adult neurogenesis 2 - Stem cells and neuronal development in the adult brain" (Oxford University Press). e-mail:gerd.kempermann@crt-dresden.de

\title{
Why and how physical activity promotes experience-induced brain plasticity
}

\author{
Gerd Kempermann ${ }^{1,2 *}$, Klaus Fabel 1,2, Dan Ehninger ${ }^{3}$, Harish Babu ${ }^{4}$, \\ Perla Leal-Galicia ${ }^{1}$, Alexander Garthe ${ }^{1}$ and Susanne A. Wolf ${ }^{5}$ \\ 1 Center for Regenerative Therapies Dresden, German Research Foundation, Dresden, Germany \\ 2 German Center for Neurodegenerative Diseases, Dresden, Germany \\ ${ }_{3}$ German Center for Neurodegenerative Diseases, Bonn, Germany \\ ${ }^{4}$ Department of Neurosurgery, Stanford University, Stanford, CA, USA \\ ${ }^{5}$ Department of Cell and Neurobiology, Institute of Anatomy, University Zürich, Zürich, Switzerland
}

Adult hippocampal neurogenesis is an unusual case of brain plasticity, since new neurons (and not just neurites and synapses) are added to the network in an activity-dependent way. At the behavioral level the plasticity-inducing stimuli include both physical and cognitive activity. In reductionistic animal studies these types of activity can be studied separately in paradigms like voluntary wheel running and environmental enrichment. In both of these, adult neurogenesis is increased but the net effect is primarily due to different mechanisms at the cellular level. Locomotion appears to stimulate the precursor cells, from which adult neurogenesis originates, to increased proliferation and maintenance over time, whereas environmental enrichment, as well as learning, predominantly promotes survival of immature neurons, that is the progeny of the proliferating precursor cells. Surprisingly, these effects are additive: boosting the potential for adult neurogenesis by physical activity increases the recruitment of cells following cognitive stimulation in an enriched environment. Why is that? We argue that locomotion actually serves as an intrinsic feedback mechanism, signaling to the brain, including its neural precursor cells, increasing the likelihood of cognitive challenges. In the wild (other than in front of a TV), no separation of physical and cognitive activity occurs. Physical activity might thus be much more than a generally healthy garnish to leading "an active life" but an evolutionarily fundamental aspect of "activity," which is needed to provide the brain and its systems of plastic adaptation with the appropriate regulatory input and feedback.

Keywords: exercise, hippocampus, reserve, learning, evolution

\section{INTRODUCTION}

A healthy mind in a healthy body is a proverbial common place but biologically poorly understood. Why and how activity is good for the brain is a question of fundamental impact for the mental health of an aging society. "Activity" falls - broadly speaking - into two major categories: physical and cognitive. Both affect brain structure and function, which has been shown in a particularly interesting case of cell based brain plasticity: adult hippocampal neurogenesis. New neurons are generated throughout life in this region providing the functional backbone for learning and memory. Recent animal and human studies have proposed the hippocampus and neurogenesis a prime target in diseases like depression and dementia.

In animal experiments, both voluntary exercise (wheel running) as well as the classical paradigm of environmental enrichment stimulate adult hippocampal neurogenesis but apparently do so 
by different means. While exercise has a strong inducing effect on precursor cell proliferation, environmental enrichment promotes the survival of newborn neurons. This leads to a simplified model implying that a generic "activity" stimulus like locomotion would induce precursor cells to divide and increase the number of cells which are potentially available for further neuronal development, whereas the more cognitive, and hence more specific stimulus resulting from the exposure to environmental complexity and novelty recruits cells from this pool of new cells for lasting functional integration. This simple model is plausible and explains many observations but actually represents only part of the full picture. First, both types of activity do have overlapping effects on adult neurogenesis and the distinction is less clear-cut than the model assumes. The reductionistic experimental paradigms might thus reduce too much of the existing complexity. But second and even more important, what has remained largely unresolved is the relationship and potential interplay between the two types of activity. The present review deals with this issue by elaborating on thoughts presented in an experimental study on the topic, published in Frontiers in Neurogenesis (Fabel et al., 2009).

\section{FUNCTIONAL AND CLINICAL RELEVANCE}

The relationship between physical and cognitive activity is not merely an academic question for scientists in biology and neuroscience deciphering the regulation of adult neurogenesis. Because of the medical implications of "activity" for mental health, adult neurogenesis has become the recent focus of many research areas, including psychology and psychiatry (Sibley and Etnier, 2003; Angevaren et al., 2008). The current concepts in the field suggest that adult hippocampal neurogenesis is central to the functionality of the dentate gyrus. New neurons add a particular level of plasticity to the network and do so in a dynamic, highly regulated manner. The newborn cells go through a period of increased synaptic plasticity, and under normal conditions, all long-term potentiation (LTP) measurable in the dentate gyrus originates from these newborn cells (Wang et al., 2000; Schmidt-Hieber et al., 2004; Saxe et al., 2006; Garthe et al., 2009). Only after a pharmacological release of the strong physiologic inhibition, the LTP of the older granule cells becomes visible. One key function of the dentate gyrus is pattern separation (Kesner, 2007). The new neurons help avoiding an interference of new memories with similar, already stored older ones in area CA3, a phenomenon referred to as catastrophic interference (Aimone et al., 2006,
2009; Wiskott et al., 2006; Appleby and Wiskott, 2009). As a consequence there appear to be lasting changes in the network due to the integration of the new neurons. Thus, the transient increased plasticity provided by adult neurogenesis leads to a lasting modification of the network. We have hypothesized that the new neurons thereby allow an activity-dependent adaptation of the mossy fiber connection between the dentate gyrus and CA3 based on previous levels of activity and experience (Kempermann, 2002). The strength of this network at this bottleneck structure would be optimized in terms of its ability to differentiate between highly similar and partially overlapping input patterns. Such ability significantly increases an animal's behavioral performance in the context of environments where non-prominent but task-specific constraints undergo substantial changes over time. An individual with a low level of activity would not only have less structural plasticity at a given time but also could not gain lasting benefit from this plasticity. And finally, as we will see, even such machinery that could provide more new neurons in the future is not prepared for such level of challenge, if previous activity has been low. One concrete benefit from the lasting network changes occurring over time might be an improved flexibility, e.g., in situations requiring the integration of novel important information into known, already experienced contexts (Garthe et al., 2009). In spatial behavioral tasks, this situation is partly revealed in so-called “reversals.” In such a reversal learning session the goal position, critical for mastering the task is changed but the more general spatial and emotional context remains stable. Changing the goal position requires the cognitive graph formed previously to be changed, too. In such a case most of a graph's nodes could remain stable, but all memories relating to the old goal position need to be updated - while kept separately from the previously learned ones, because they have been proven to be effective in guiding the animal's behavior. Despite positive results gained from studies assessing the short-term effects of adult neurogenesis, those specifically targeting long-term benefits are as yet lacking.

In any case, although currently no unifying theory exists about the function of adult hippocampal neurogenesis, there can be little doubt that the new neurons do contribute to the functionality of the dentate gyrus. Consequently, failing function of the hippocampus and the dentate gyrus has been linked to a number of disorders, most notably dementias as well as major depression and schizophrenia (Jacobs et al., 2000; Santarelli et al., 2003; Reif et al., 2007; Sahay and Hen, 2007). 


\footnotetext{
Enriched environment

Enriched environments are "the complex combination of social and inanimate stimulation" (Rosenzweig and Bennett, 1996) and consist of a large group of animals living in a big cage with exchangeable tunnels, bridges, and toys. This condition is usually compared to the rather Spartan conditions of regular laboratory housing.
}

Given the increasing evidence for a causal link between adult hippocampal neurogenesis and functionality, it is only a small step to the idea that disturbed adult neurogenesis might explain at least the hippocampal aspects of the pathology in these cases. Finally, and most relevant for the present context: in all three disorders clinical evidence suggests that the individuals level of overall activity, either physical or cognitive, at least partly counteracts the impairment (Wilson et al., 2002; Sagatun et al., 2007; Daley, 2008; Scarmeas et al., 2009; Pajonk et al., 2010). We thus propose that this beneficial, largely preventive and partly also restorative effect of activity can to a relevant degree be explained by the activity-dependent functional contribution of adult-born neurons.

\section{ENRICHED ENVIRONMENTS}

The classical model to study the impact of "experience" on the brain in animal studies is "enriched environment." In the context of studying the principles underlying basic rules of learning Donald Hebb explored how early experience influenced cognitive abilities later in life. He compared rats in standard laboratory cages with rats he had taken home and found long-lasting positive effects on learning and memory (Hebb, 1947; Hymovitch, 1952).

Research on the effects of "enriched environments," in the late 1950s to the mid-1970s became a central paradigm in developmental psychology because it allowed researchers to experimentally address fundamental questions such as the relative contributions of inheritance and education? From a biological point of view this is largely equivalent to asking for the respective contribution of genes and environment to development. The genetic influence can, for example, be kept constant by examining inbred strains of mice or rats. Molecular biology and genetic research allow the direct study of the interaction between genes and environment, together constituting developmental plasticity.

Mark Rosenzweig and E. L. Bennett and their colleagues published an impressive body of studies beginning in the 1960s in which they showed the wide range and dynamic changes in numerous measures describing the brain of animals exposed to environmental enrichment (Bennett, 1976; Rosenzweig and Bennett, 1996; Rosenzweig, 2003). Animals living in an enriched environment are compared with animals living under the usual, rather plain conditions of laboratory housing. This simple manipulation has far-reaching effects on the brain and its function. Most notably, they made the conceptual link between "activity," or experience, and gross alterations in brain structure. They showed that environmental enrichment profoundly and broadly influences the anatomy and physiology of the brain.

In contrast to common belief, environmental enrichment is more than just a reversal of otherwise prevailing impoverishment of laboratory housing. Although environmental enrichment brings laboratory animals closer to feral conditions, it provides animals, as Lamberto Maffei and colleagues have pointed out, with "a kind of challenge-free interaction with a stimulating surrounding." And they go on: "We might speculate that, although the activity of mice and rats in the wild is mostly driven by necessity, in an enriched environment it is usually prompted by a combination of curiosity and play" (Sale et al., 2009). The important point here is that enriched environments are poor mirrors of the feral conditions. An enriched environment is a designed world, aiming at providing a particular, mostly cognitive challenge that is also relevant to living in the wild but this would be embedded into a larger context. As we will see below, we believe that one of the key contextual parameters that is lacking in the laboratory situation is the amount of physical activity that is demanded from an animal in the wild. This does not necessarily and per se alter the parameters of interest: for example, highly similar levels of adult hippocampal neurogenesis have been found in wild vs. laboratory-bred rats of different strains (Epp et al., 2009).

In 1997 we reported that mice living in an enriched environment had more new hippocampal granule cells than controls (Kempermann et al., 1997). Although in a still rather loose way, the positive regulation of adult neurogenesis was thereby linked to hippocampal function. Cognitive activation "made" new neurons. This pro-neurogenic effects of environmental enrichment was even maintained into old age, where from an extremely low baseline level of adult neurogenesis the relative increase was even larger than in younger animals (Kempermann et al., 1998b, 2002).

Although some as yet unexplored strain differences (and hence genetic variation) exist (Kempermann et al., 1998a), this effect was due to a survival-promoting effect on the progeny of the dividing precursor cells but not to increased divisions of the precursor cells (Brandt et al., 2003; Kronenberg et al., 2003; Steiner et al., 2008). Nevertheless, over longer periods of time, environmental enrichment affected the number of precursor cells (Kronenberg et al., 2003), resulting in an apparent increase in cell proliferation in long-term experiments (Kempermann and Gage, 1999; Kempermann et al., 2002). 
Historically, most enriched environments contained running wheels, blurring the distinction between the two paradigms. Only direct comparison of both paradigms reveals how different they actually are. This has not been explored for many questions, but in the case of adult neurogenesis the differences are striking. Physical activity alone certainly does not explain the effects of environmental enrichment (van Praag et al., 1999b; Kronenberg et al., 2003; Steiner et al., 2008).

\section{A COMPLEXITY AND NOVELTY HYPOTHESIS OF ENVIRONMENTAL ENRICHMENT}

Environmental enrichment arguably represents a complex set of cognitive (and possibly affective) stimuli. Much in the spirit of the theory-driven 1970s a "developmental" theory of environmental enrichment has sometimes been contrasted with the "learning theory" favored by Rosenzweig. The two theories, however, do not necessarily target the same aspects. In fact, R.A. Cummins emphasis on development aimed at answering the question, why and how the response to enrichment changes with developmental stage (Cummins et al., 1977).

The relevant key stimuli might be complexity and novelty (Kempermann, 2002; Kempermann et al., 2004), which are experienced by free exploration. For mice and rats living in the wild, this exploration is intricately linked to physical activity. They have to move through their world in their search for food, mates, and shelter. The reductionist setting of the experimental enriched environment (without running wheels) largely strips the locomotive aspect from the experience of complexity and novelty. Critically, the changes occurring in an enriched environment add a degree of uncertainty to the life of the animal. Watters (2009), approaching the question from the perspective of keeping animals in zoos, has suggested that according to learning theory the motivation of the animal "to perform" is best maintained when reward is uncertain and that consequently, the best enriched environment is one of dosed uncertainty. Uncertainty contains a component of novelty, which is a powerful cognitive stimulus for laboratory animals as for humans. In the olfactory bulb, exposure to novelty (by exposing mice to novel odorants) appears to be a key regulator of olfactory neurogenesis (Veyrac et al., 2009). Enhanced responsiveness to novel events in aged humans (i.e., the duration and size of the P3 event-related potential in the EEG), was linked to improved performance in neuropsychological tests, in particular those containing attention/executive functions (Daffner et al., 2006).
Our enriched environments have not been optimized for the aspects of uncertainty and novelty but it is clear that in terms of predictability they are less stable than standard housing conditions. The environmental enrichments used do not simply provide more space - they generally allow the expression of a much wider range of spatial and social behaviors. The increased spatial complexity allows for an increased exploratory behavior, learning about new objects and configurations or display of more naturalistic behavioral patterns such as marking, sheltering and defense of territories. Thus, despite being exposed to a highly controlled, artificial situation rodents living in an environmental enrichment develop social hierarchies with all accompanying behaviors like aggression, defense and social avoidance. Together, this spatial and social context provides lots of opportunities for learning associations between places, social rules, and events, all of which having a strong hippocampal component. If learning is coping with novelty and change over time, the experience of an enriched environment grants a variety of opportunities for continued learning (Greenough, 1976; Renner and Rosenzweig, 1987).

Consistent with this idea, learning stimuli such as training in the Morris water maze had similar survival-promoting effects on adult hippocampal neurogenesis as environmental enrichment and did so during particular critical time windows, roughly at the time when the new neurons had made dendritic and axonal connections and lasting for 2-3 weeks (Gould et al., 1999; Leuner et al., 2004). It is not clear, if and how environmental enrichment affects even later stages of neuronal development, after the cells have become fully functional. At that advanced stage a similar survival-promoting effect has been proposed and has been described under particular experimental situations, (Tozuka et al., 2005; Ge et al., 2007; Kee et al., 2007; Tashiro et al., 2007) but has not yet been unambiguously confirmed.

In yet other experiments, however, even the early learning-induced survival effects were not observed (van Praag et al., 1999b; Ambrogini et al., 2004; Ehninger and Kempermann, 2006). Enriched environments affect adult neurogenesis also well before the new neurons are integrated (Steiner et al., 2008). Although there are clear differences in the experimental design between these studies, in particular with respect to a sensitive period of the new neurons, the question of whether learning per se can directly recruit new neurons into function in the end remains open. In favor of this hypothesis is the observation that the induction of LTP as the presumed 
electrophysiological correlate of learning induces adult hippocampal neurogenesis in vitro (Babu et al., 2009) and in vivo (Bruel-Jungerman et al., 2006).

\section{THE NEUROGENIC MAINTENANCE EFFECT OF ENVIRONMENTAL ENRICHMENT}

The recruitment effect of environmental enrichment draws from a pool of potentially recruitable cells. These are primarily the early postmitotic neurons. But if applied over prolonged periods of time, environmental enrichment had additional effects on the precursor cells (Kempermann and Gage, 1999), consistent with the idea that the precursor cells themselves can sense neuronal activity and translate it into regulation of adult neurogenesis (Deisseroth et al., 2004; Babu et al., 2009). Precursor cell proliferation was maintained at a higher level than in controls. Indeed, short-term experiments showed that even then, environmental enrichment affects precursor cells, mostly at the more advanced and neuronally determined level (Steiner et al., 2008). This all suggests that somehow the machinery underlying adult neurogenesis at the precursor cell level "learns" how much activity to expect and to adjust the level of available proliferating precursor cells. General features of "activity" might be a straightforward way to relate this information, although more complex feedback mechanisms from the more advanced levels of neuronal development to the precursor cells are conceivable.

\section{VOLUNTARY WHEEL RUNNING}

Laboratory rodents make extensive use of a running wheel in their cage and run as much as 5-8 km a night, probably equivalent to their distances covered in the wild (van Praag et al, 1999a; Chappell et al, 2004). Generally, voluntary physical activity has been a very robust stimulus for adult hippocampal neurogenesis in rodents from birth to oldest age (van Praag et al., 1999a,b, 2005; Kronenberg et al., 2003; Bick-Sander et al., 2006; Steiner et al., 2008; Wu et al., 2008; Kannangara et al., 2010). Running can at least in part reverse the massive decrease in neurogenesis observed in aged animals (Kronenberg et al., 2006). Furthermore, running improves learning (as detected in the water maze task) in aged animals (van Praag et al., 2005). The fact that wild mice did not show an increase in adult neurogenesis after exposure to a running wheel in the laboratory (Hauser et al., 2009) might simply reflect a ceiling effect. Running acutely increases precursor cell proliferation at the level of the type- 2 progenitor cells and this short-term effect wears off over a number of weeks (Kronenberg et al., 2003; Steiner et al., 2008). In addition, there is a linked effect on the consecutive stages of neuronal development and also a survival-promoting effect, especially over prolonged periods of training (Kronenberg et al., 2006; Snyder et al., 2009). Long-term running maintained precursor cell proliferation at an elevated level. Presumably in the absence of appropriate survival-promoting stimuli, however, this increased potential was not translated into an increase in net neurogenesis. This suggested that physical activity is a necessary but not sufficient mechanism to sustain adult neurogenesis in the long run. There is an activity-dependent component in the regulation of adult hippocampal neurogenesis that is not related to physical exercise itself, because circadian phase, and thus activity in a more general sense also correlates with levels of adult hippocampal neurogenesis (Holmes et al., 2004). The effects on adult neurogenesis cannot be distinguished from those of environmental enrichment in a clear-cut manner: depending on the time-scales of the experiments the two paradigms show different degrees of overlap in their effect on adult neurogenesis. But more importantly, they show distinct differences, in particular when compared side-by-side.

When rats ambulate, this has an impressive, very direct effect on LTP as the electrophysiological correlate of learning (Leung et al., 2003). And in wheel-running mice LTP was also increased (van Praag et al., 1999a). Theta rhythms triggered by repetitive motor movements, might underlie this finding. The direct link between theta waves (or other endogenous oscillations) and the regulation of adult neurogenesis has not yet been made but the cholinergic input from the medial septal area, which is known to regulate hippocampal theta promotes precursor cell proliferation in the dentate gyrus (Cooper-Kuhn et al., 2004; Itou et al., 2010). In addition, in co-cultures of precursor cells with hippocampal neurons, chemical induction of LTP (by adding glycine in magnesium-free media) caused oscillating synchronous synaptic activity in the neurons, which in turn induced the precursor cells to differentiate into neurons (Babu et al., 2009). In line with this consideration, neuronal activity and neurotransmitter signaling through, most notably $N$-methyl-D-aspartate (NMDA) receptors, might mediate at least part of the neurogenesis effect of physical activity. At least in cell culture, NMDA dependent pathways are involved in the activity-dependent control of adult neurogenesis (Deisseroth et al., 2004; Babu et al., 2009). In vivo studies with NMDA antagonists point into the same direction (Nacher and McEwen, 2006; Nacher et al., 2007; Petrus et al., 2008). 
The idea of neural or cognitive reserves The neural reserve is the range of compensation that a brain has to functionally compensate impairments due to disease or trauma. The theories were first devised to account for the fact that for example in cases of dementia no simple correlation exists between the amount of neuropathological signs and the degree of cognitive impairment.

The neurogenic reserve

The idea of the neurogenic reserve takes up the "neural reserve hypothesis" in the context of adult neurogenesis and maintains that the ability to generate new hippocampal neurons provides a potential for lifelong cognitive flexibility and adaptability.

\section{THE EFFECTS OF PHYSICAL ACTIVITY AND ENVIRONMENTAL ENRICHMENT ARE ADDITIVE}

Sequentially combining the potential-increasing effects of physical activity with the survival promoting effect of environmental enrichment revealed that the two effects are indeed additive (Fabel et al., 2009; Figure 1). A stimulated potential yielded even more new neurons than the baseline potential despite the fact that only a subset of new cells survives.

The important consequence of this latter observation is that the size of the potential somehow seems to determine the extent of the net effect at the end of the day. It thus seems that although proliferation is a poor predictor of net neurogenesis, maintenance of proliferating precursor cells is a good way of obtaining a larger neurogenic response to the same stimulus. Whether or not such "larger neurogenic response" is indeed also functionally more useful remains to be shown, but as function triggers the recruitment, such assumption can at least be safely made.

The experiment confirmed two previous observations. First, 5 weeks after cessation of exercise, neurogenesis from precursor cells activated during the last days of running was elevated. This tells us that there is indeed a proneurogenic effect of exercise, even if no specific stimulus is added. Second, because proliferating cells were labeled before animals entered the enriched environment, the increase in adult neurogenesis after enrichment was confirmed to be a true survival effect. No previous nonspecific activation (beyond the stimuli present in the control condition of regular laboratory housing) is necessary.

\section{THE "NEUROGENIC RESERVE HYPOTHESIS"}

Obviously, activity cannot rescue progeny from precursor cells that have already been lost. Precursor cells can only be replaced up to the proliferative potential of the remaining cells. The difficult key experiment to do would be a repopulation study to tease out the limits of this process. Even though at old age the activity-induced proliferation of precursor cells was much greater than at young age, the overall absolute level was much lower (Kempermann et al., 1998b, 2002). Continued stimulation beginning early, in contrast, maintained the potential but is obviously dependent on the level of precursor cell proliferation (and the number of precursor cells) at the time when training starts. The consequence from this observation is that a lack of activity early in life can only be partly compensated later.

Our hypothesis is that continued activity maintains the potential for adult neurogenesis and thereby creates a "neurogenic reserve" that is a pool of potentially recruitable neurons that would allow a sustained adaptability of the hippocampal network (Kempermann, 2008). Previous experience and activity level would be used as predictor for levels of experience and activity to be expected in the future. The neurogenic reserve would be optimized in size to the amount of novelty and complexity that an animal is likely to experience. This optimization process would suggest that the expensive machinery for adult neurogenesis is only maintained if there is likelihood that it is needed. The finding that the effects of exercise and enrichment on adult neurogenesis are additive is direct experimental evidence in support of the "neurogenic reserve hypothesis." Obviously, though, more research needs to be done. A longterm experiment would have to show a similar

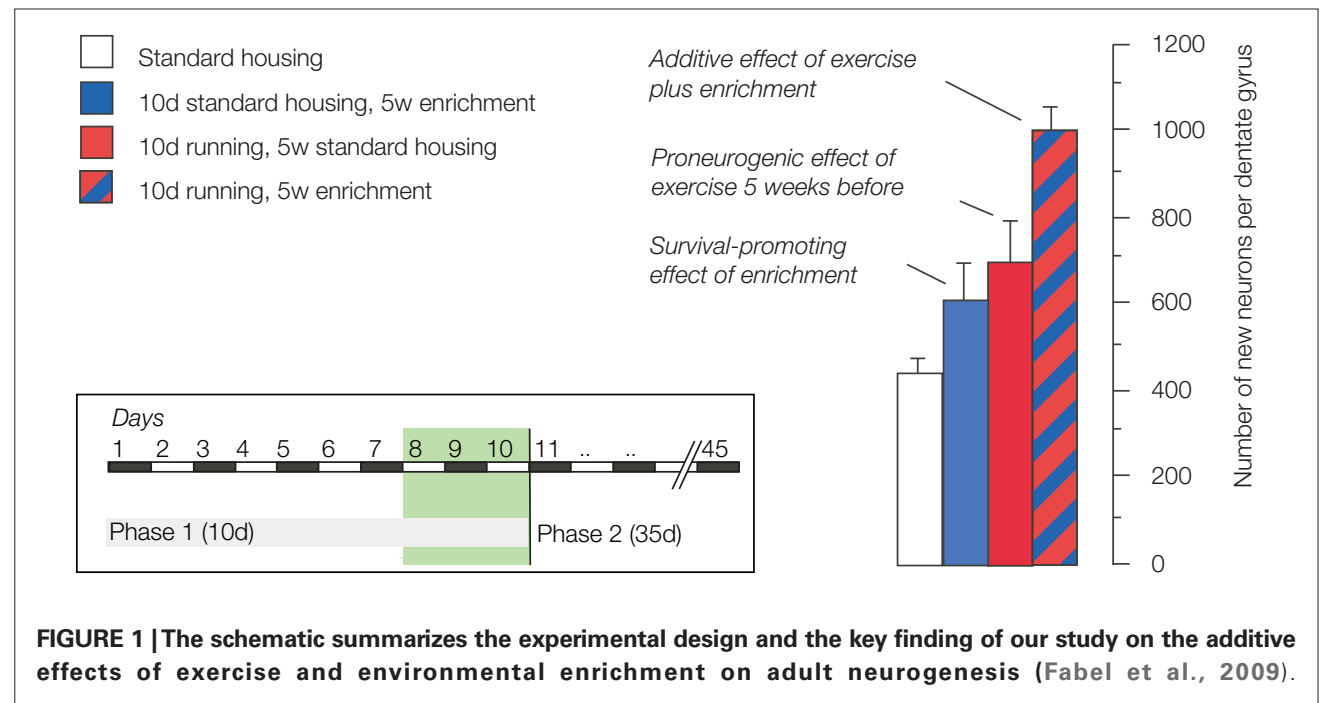


effect on much longer time-scales, and the concrete functional outcome of this manipulation needs to be addressed. Finally, one would have to show that the early reserve-building intervention is indeed superior to later attempts for traininginduced compensation.

\section{ATTEMPT OF A SYNTHESIS}

In animals, most if not all aspects of cognition are inseparable from locomotion and physical activity. Exploration, spatial navigation, and most types of learning accessible in a rodent are based on its movement in the outer world. Search for food, shelter, and mates are physical activities, requiring mental input to be successful on both a phylogenetic and ontogenetic scale. Consequently, the fact that running induces neurogenesis will be less counterintuitive if one appreciates physical activity as a basis for cognition. This idea is also in line with other hypotheses developed for foodcaching birds (Grodzinski and Clayton, 2010) and squirrels (Johnson et al., 2010), in which the navigational challenge is interpreted as one key stimulus for the development (and evolution) of particular cognitive traits.

An important question is, whether this association is preserved in humans. Several epidemiological and intervention studies suggest so (Sibley and Etnier, 2003; Angevaren et al., 2008). Among them a study with the incredible $N$ of 1.2 million: in conscripts for the Swedish military, cardiovascular fitness (but not muscle strength) correlated significantly with intelligence (Aberg et al., 2009). There is also direct evidence now that physical exercise results in measurable changes in hippocampal volume (Erickson et al., 2009; Pajonk et al., 2010), but adult neurogenesis obviously could not be studied in humans.

There is no linear dose effect in the effect of running on adult neurogenesis: although in acute settings more running led to more proliferation, even low amounts of exercise induced cell proliferation (Holmes et al., 2004). We had speculated in that study that activity "per se" would be sufficient to lead to some induction. One study even suggested that the presence of the running wheel alone had already some effect (Bednarczyk et al., 2010). In line with that research is the suggestion that size of the territory of an animal does not seem to show a good correlation with the level of adult neurogenesis (assuming that larger territories would require more locomotion; Amrein et al., 2004, 2007). See also related discussions in Barker et al. (2005) and Amrein et al. (2008).

The artificial separation of exercise and enrichment is experimentally useful and meaningful but distracts from the fact that both are in fact inseparable. All brain output is motoric but input and output structures are linked closer than the concept of "associative areas" in the brain suggested. Consequently, the link between sensory and motor activity ultimately takes place at the level of single neurons. Research on adult neurogenesis adds a new facet to this insight by demonstrating that something similar also occurs in adult hippocampal precursor cells and their progeny.

Physical activity would be an intrinsic behavior-based signal to the brain (and hippocampus) implying that the likelihood of cognitive challenge is increased. In addition, one could speculate that running long distances increases the chance to encounter new environments increasing the need for spatial orientation and memory like wild animals that need to find the way back to their safe shelter. In the wild, physical movement through environments is a necessary prerequisite for spatial exploration and, hence, the establishment of spatial representations encoded in the hippocampus (e.g., place cells). Accordingly, from the point of view of spatial learning, physical activity is required for providing relevant sensory information to the animal that is then used to construct a representation of the environment in the hippocampus.

Despite being reductionistic "enriched environments" are immensely complex experimental paradigms. To isolate the contribution of physical activity is comparatively straightforward. It remains a far greater challenge to unravel the role of other factors such as social interaction, novelty, stress, etc., within that complexity.

The hippocampus would use physical activity and locomotion as surrogate indicator of cognitive demand rather than relying on analyzing the density and probability of cognitive stimuli themselves. Physical activity as a measure of overall brain activity is a very simple way of converting environmental complexity and assessment of the need for cognition into a plain biological parameter that - one is inclined to say - even a stem cell can understand.

If further confirmed, this idea has obvious implications for our judgment of which kind of activity we deem useful or "healthy." If physical activity and its consequences are evolutionarily inseparable from cognition, training to improve cognition will inevitably benefit from, if not depend on physical exercise.

\section{ACKNOWLEDGMENTS}

This study was supported by Volkswagenstiftung. Perla Leal-Galicia was supported by Alexander von Humboldt Foundation. 


\section{REFERENCES}

Aberg, M. A., Pedersen, N. L., Toren, K., Svartengren, M., Backstrand, B., Johnsson, T., Cooper-Kuhn, C. M., Aberg, N. D., Nilsson, M., and Kuhn, H. G. (2009). Cardiovascular fitness is associated with cognition in young adulthood. Proc. Natl. Acad. Sci. U.S.A. 106, 20906-20911.

Aimone, J. B., Wiles, J., and Gage, F. H. (2006). Potential role for adult neurogenesis in the encoding of time in new memories. Nat. Neurosci. 9 , 723-727.

Aimone, J. B., Wiles, J., and Gage, F. H. (2009). Computational influence of adult neurogenesis on memory encoding. Neuron 61, 187-202.

Ambrogini, P., Orsini, L., Mancini, C., Ferri, P., Ciaroni, S., and Cuppini, R. (2004). Learning may reduce neurogenesis in adult rat dentate gyrus. Neurosci. Lett. 359, 13-16.

Amrein, I., Dechmann, D. K., Winter, Y., and Lipp, H. P. (2007). Absent or low rate of adult neurogenesis in the hippocampus of bats (Chiroptera). PLoS ONE 2, e455. doi: 10.1371/journal. pone.0000455.

Amrein, I., Lipp, H. P., Boonstra, R., and Wojtowicz, J. M. (2008). "Adult hippocampal neurogenesis in natural populations of mammals," in Adult Neurogenesis, eds F. H. Gage, G. Kempermann, and H. Song (Cold Spring Harbor, NY: Cold Spring Harbor Laboratory Press), 645-659.

Amrein, I., Slomianka, L., Poletaeva, II, Bologova, N.V., and Lipp, H. P. (2004). Marked species and age-dependent differences in cell proliferation and neurogenesis in the hippocampus of wild-living rodents. Hippocampus 14 , 1000-1010.

Angevaren, M., Aufdemkampe, G., Verhaar, H. J., Aleman, A., and Vanhees, L. (2008). Physical activity and enhanced fitness to improve cognitive function in older people without known cognitive impairment. Cochrane Database Syst. Rev. CD005381.

Appleby, P. A., and Wiskott, L. (2009), Additive neurogenesis as a strategy for avoiding interference in a sparsely-coding dentate gyrus. Network 20, 137-161.

Babu, H., Ramirez-Rodriguez, G., Fabel, K., Bischofberger, J., and Kempermann, G. (2009). Synaptic network activity induces neuronal differentiation of adult hippocampal precursor cells through BDNF signaling. Front. Neurosci. 3:49. doi: 10.3389/ neuro.22.001.2009.

Barker, J. M., Wojtowicz, J. M., and Boonstra, R. (2005). Where's my dinner? Adult neurogenesis in free-living food-storing rodents. Genes Brain Behav. 4, 89-98.

Bednarczyk, M. R., Hacker, L. C., FortinNunez, S., Aumont, A., Bergeron, R. and Fernandes, K. J. (2010). Distinct stages of adult hippocampal neurogenesis are regulated by running and the running environment. Hippocampus PMID: 20623741 [Epub ahead of print].

Bennett, E. L. (1976). "Cerebral effects of differential experience and training," in Neural Mechanisms of Learning and Memory, eds M. R. Rosenzweig and E. L. Bennett (Cambridge: MIT Press), 279-287.

Bick-Sander, A., Steiner, B., Wolf, S. A., Babu, H., and Kempermann, G. (2006). Running in pregnancy transiently increases postnatal hippocampal neurogenesis in the offspring. Proc. Natl. Acad. Sci. U.S.A. 103 3852-3857.

Brandt, M. D., Jessberger, S., Steiner, B. Kronenberg, G., Reuter, K., BickSander, A., von der Behrens, W., and Kempermann, G. (2003). Transient calretinin expression defines early postmitotic step of neuronal differentiation in adult hippocampal neurogenesis of mice. Mol. Cell. Neurosci. 24, 603-613.

Bruel-Jungerman, E., Davis, S., Rampon, C., and Laroche, S. (2006). Long-term potentiation enhances neurogenesis in the adult dentate gyrus. J. Neurosci. 26, 5888-5893.

Chappell,M.A., Garland, T. Jr., Rezende, E. L., and Gomes, F. R. (2004 ). Voluntary running in deer mice: speed, distance, energy costs and temperature effects. J Exp Biol. 207, 3839-3854.

Cooper-Kuhn, C. M., Winkler, J., and Kuhn, H. G. (2004). Decreased neurogenesis after cholinergic forebrain lesion in the adult rat. J. Neurosci. Res. $77,155-165$.

Cummins, R. A., Livesey, P. J., and Evans, J. G. (1977). A developmental theory of environmental enrichment. Science 197, 692-694.

Daffner, K. R., Ryan, K. K., Williams, D. M., Budson, A. E., Rentz, D. M. Wolk, D. A., and Holcomb, P. J. (2006). Increased responsiveness to novelty is associated with successful cognitive aging. J. Cogn. Neurosci. 18, 1759-1773.

Daley, A. (2008). Exercise and depression: a review of reviews. J. Clin. Psychol. Med. Settings 15, 140-147.

Deisseroth, K., Singla, S., Toda, H., Monje, M., Palmer, T. D., and Malenka, R. C. (2004). Excitation-neurogenesis coupling in adult neural stem/progenitor cells. Neuron 42, 535-552.

Ehninger, D., and Kempermann, G. (2006). Paradoxical effects of learning the Morris water maze on adult hippocampal neurogenesis in mice may be explained by a combination of stress and physical activity. Genes Brain Behav. 5, 29-39.

Epp, J. R., Barker, J. M., and Galea, L. A (2009). Running wild: neurogenesis in the hippocampus across the lifespan in wild and laboratory-bred Norway rats. Hippocampus 19, 1040-1049.

Erickson, K. I., Prakash, R. S., Voss, M. W., Chaddock, L., Hu, L., Morris, K. S., White, S. M., Wojcicki, T. R. McAuley, E., and Kramer, A. F. (2009) Aerobic fitness is associated with hippocampal volume in elderly humans. Hippocampus 19, 1030-1039.

Fabel, K., Wolf, S. A., Ehninger, D., Babu, H., Galicia, P. L., and Kempermann, G. (2009). Additive effects of physical exercise and environmental enrichment on adult hippocampal neurogenesis in mice. Front. Neurosci. 3, 50 doi: 10.3389/neuro.22.002.2009.

Garthe, A., Behr, J., and Kempermann, G. (2009).Adult-generated hippocampal neurons allow the flexible use of spatially precise learning strategies. PLoS ONE 4, e5464. doi: 10.1371/journal. pone. 0005464

Ge, S., Yang, C. H., Hsu, K. S., Ming, G. L., and Song, H. (2007). A critical period for enhanced synaptic plasticity in newly generated neurons of the adult brain. Neuron 54, 559-566.

Gould, E., Beylin, A., Tanapat, P., Reeves, A., and Shors, T. J. (1999). Learning enhances adult neurogenesis in the hippocampal formation. Nat. Neurosci. 2, 260-265.

Greenough, W. T. (1976). "Enduring brain effects of differential experience and training," in Neural Mechanisms of Learning and Memory, eds M. R. Rosenzweig and E. L. Bennett (Cambridge: MIT Press), 255-278.

Grodzinski, U., and Clayton, N. S. (2010), Problems faced by food-caching corvids and the evolution of cognitive solutions. Philos. Trans. R. Soc. Lond. B, Biol. Sci. 365, 977-987.

Hauser, T., Klaus, F., Lipp, H. P., and Amrein, I. (2009). No effect of running and laboratory housing on adult hippocampal neurogenesis in wild caught long-tailed wood mouse. $B M C$ Neurosci. 10, 43 .

Hebb, D. O. (1947). The effects of early experience on problem-solving at maturity. Am. Psychol. 2, 306-307.

Holmes, M. M., Galea, L. A., Mistlberger, R. E., and Kempermann, G. (2004) Adult hippocampal neurogenesis and voluntary running activity: circadian and dose-dependent effects. J. Neurosci. Res. 76, 216-222.

Hymovitch, B. (1952). The effects of experimental variation on problem solving in the rat. J. Comp. Physiol. Psychol. 45, 313-321.
Itou, Y., Nochi, R., Kuribayashi, H., Saito, Y., and Hisatsune, T. (2010) Cholinergic activation of hippocampal neural stem cells in aged dentate gyrus. Hippocampus PMID: 20054812 [Epub ahead of print].

Jacobs, B. L., Praag, H., and Gage, F. H. (2000). Adult brain neurogenesis and psychiatry: a novel theory of depression. Mol. Psychiatry 5, 262-269.

Johnson, K. M., Boonstra, R., and Wojtowicz,J.M. (2010).Hippocampal neurogenesis in food-storing red squirrels: the impact of age and spatial behavior. Genes Brain Behav. 9, 583-591.

Kannangara, T. S., Lucero, M. J., GilMohapel, J., Drapala, R. J., Simpson, J. M., Christie, B. R., and van Praag, H. (2010). Running reduces stress and enhances cell genesis in aged mice. Neurobiol. Aging doi:10.1016/j. neurobiolaging.2009.12.025 [Epub ahead of print].

Kee, N., Teixeira, C. M., Wang, A. H., and Frankland, P. W. (2007). Preferential incorporation of adult-generated granule cells into spatial memory networks in the dentate gyrus. Nat. Neurosci. 10, 355-362.

Kempermann, G. (2002). Why new neurons? Possible functions for adult hippocampal neurogenesis. J. Neurosci. 22, 635-638.

Kempermann, G. (2008). The neurogenic reserve hypothesis: what is adult hippocampal neurogenesis good for? Trends Neurosci. 31, 163-169.

Kempermann, G., Brandon, E. P., and Gage, F. H. (1998a). Environmental stimulation of 129/SvJ mice causes increased cell proliferation and neurogenesis in the adult dentate gyrus. Curr. Biol. 8, 939-942.

Kempermann, G., Kuhn, H. G., and Gage, F. H. (1998b). Experience-induced neurogenesis in the senescent dentate gyrus. J. Neurosci. 18, 3206-3212.

Kempermann, G., and Gage, F. H. (1999). Experience-dependent regulation of adult hippocampal neurogenesis: effects of long-term stimulation and stimulus withdrawal. Hippocampus 9, 321-332.

Kempermann, G., Gast, D., and Gage, F. H. (2002). Neuroplasticity in old age: sustained fivefold induction of hippocampal neurogenesis by longterm environmental enrichment. Ann. Neurol. 52, 135-143.

Kempermann, G., Kuhn, H. G., and Gage, F. H. (1997). More hippocampal neurons in adult mice living in an enriched environment. Nature 386, 493-495.

Kempermann, G., Wiskott, L., and Gage, F. H. (2004). Functional significance of adult neurogenesis. Curr. Opin. Neurobiol. 14, 186-191. 
Kesner, R. P. (2007). A behavioral analysis of dentate gyrus function. Prog. Brain Res. 163, 567-576.

Kronenberg, G., Bick-Sander, A., Bunk, E., Wolf, C., Ehninger, D., and Kempermann, G. (2006). Physical exercise prevents age-related decline in precursor cell activity in the mouse dentate gyrus. Neurobiol. Aging 27, 1505-1513.

Kronenberg, G., Reuter, K., Steiner, B., Brandt, M. D., Jessberger, S., Yamaguchi, M., and Kempermann, G. (2003). Subpopulations of proliferating cells of the adult hippocampus respond differently to physiologic neurogenic stimuli. J. Comp. Neurol. 467, 455-463.

Leuner, B., Mendolia-Loffredo, S., Kozorovitskiy, Y., Samburg, D., Gould, E., and Shors, T. J. (2004). Learning enhances the survival of new neurons beyond the time when the hippocampus is required for memory. J. Neurosci. 24, 7477-7481.

Leung, L. S., Shen, B., Rajakumar, N., and Ma, J. (2003). Cholinergic activity enhances hippocampal long-term potentiation in CA1 during walking in rats. J. Neurosci. 23, 9297-9304.

Nacher, J., and McEwen, B. S. (2006). The role of $\mathrm{N}$-methyl-D-aspartate receptors in neurogenesis. Hippocampus $16,267-270$.

Nacher, J., Varea, E., Miguel Blasco-Ibanez, J., Gomez-Climent, M. A., CastilloGomez, E., Crespo, C., MartinezGuijarro, F. J., and McEwen, B. S. (2007). N-methyl-D-aspartate receptor expression during adult neurogenesis in the rat dentate gyrus. Neuroscience 144, 855-864.

Pajonk, F. G., Wobrock, T., Gruber, O., Scherk, H., Berner, D., Kaizl, I., Kierer, A., Muller, S., Oest, M., Meyer, T., Backens, M., Schneider-Axmann, T., Thornton, A. E., Honer, W. G., and Falkai, P. (2010). Hippocampal plasticity in response to exercise in schizophrenia. Arch. Gen. Psychiatry 67, 133-143.

Petrus, D. S., Fabel, K., Kronenberg, G., Winter, C., Steiner, B., and Kempermann, G. (2008). NMDA and benzodiazepine receptors have synergistic and antagonistic effects on precursor cells in adult hippocampal neurogenesis. Eur. J. Neurosci. 29, 244-252.

Reif, A., Schmitt, A., Fritzen, S., and Lesch, K. P. (2007). Neurogenesis and schizophrenia: dividing neurons in a divided mind? Eur. Arch. Psychiatry Clin. Neurosci. 257, 290-299.

Renner, M. J., and Rosenzweig, M. R. (1987). Enriched and Impoverished Environments - Effects on Brain and Behavior. New York: Springer.

Rosenzweig, M. R. (2003). Effects of differential experience on the brain and behavior. Dev. Neuropsychol. 24, 523-540.

Rosenzweig, M. R., and Bennett, E. L. (1996). Psychobiology of plasticity: effects of training and experience on brain and behavior. Behav. Brain Res. 78, 57-65.

Sagatun, A., Sogaard, A. J., Bjertness, E., Selmer, R., and Heyerdahl, S. (2007). The association between weekly hours of physical activity and mental health: a three-year follow-up study of 15-16-year-old students in the city of Oslo, Norway. BMC Public Health 7, 155 .

Sahay, A., and Hen, R. (2007). Adult hippocampal neurogenesis in depression. Nat. Neurosci. 10, 1110-1115.

Sale, A., Berardi, N., and Maffei, L. (2009). Enrich the environment to empower the brain. Trends Neurosci. 32, 233-239.

Santarelli, L., Saxe, M., Gross, C., Surget, A., Battaglia, F., Dulawa, S., Weisstaub, N., Lee, J., Duman, R., Arancio, O., Belzung, C., and Hen, R. (2003). Requirement of hippocampal neurogenesis for the behavioral effects of antidepressants. Science 301, 805-809.

Saxe, M. D., Battaglia, F., Wang, J. W., Malleret, G., David, D. J., Monckton, J. E., Garcia, A. D., Sofroniew, M. V., Kandel, E. R., Santarelli, L., Hen, R., and Drew, M. R. (2006). Ablation of hippocampal neurogenesis impairs contextual fear conditioning and synaptic plasticity in the dentate gyrus. Proc. Natl. Acad. Sci. U.S.A. 103, 17501-17506.

Scarmeas, N., Luchsinger, J.A., Schupf, N., Brickman, A. M., Cosentino, S., Tang, M. X., and Stern, Y. (2009). Physical activity, diet, and risk of Alzheime disease. JAMA 302, 627-637.

Schmidt-Hieber, C., Jonas, P., and Bischofberger, J. (2004). Enhanced synaptic plasticity in newly generated granule cells of the adult hippocampus. Nature 429, 184-187.

Sibley, B. A., and Etnier, J. L. (2003). The relationship between physical activity and cognition in children: a meta-analysis. Pediatr. Exerc. Sci. 15, 243-256.

Snyder, J. S., Glover, L. R., Sanzone, K. M., Kamhi, J. F., and Cameron, H. A. (2009). The effects of exercise and stress on the survival and maturation of adult-generated granule cells. Hippocampus 19, 898-906.

Steiner, B., Zurborg, S., Horster, H., Fabel, K., and Kempermann, G. (2008). Differential $24 \mathrm{~h}$ responsiveness of Prox1-expressing precursor cells in adult hippocampal neurogenesis to physical activity, environmental enrichment, and kainic acid-induced seizures. Neuroscience 154, 521-529.

Tashiro, A., Makino, H., and Gage, F. H. (2007). Experience-specific functional modification of the dentate gyrus through adult neurogenesis: a critical period during an immature stage. J. Neurosci. 27, 3252-3259.

Tozuka, Y., Fukuda, S., Namba, T., Seki, T. and Hisatsune, T. (2005). GABAergic excitation promotes neuronal differentiation in adult hippocampal progenitor cells. Neuron 47, 803-815.

van Praag, H., Christie, B. R., Sejnowski, T. J., and Gage, F. H. (1999a). Running enhances neurogenesis, learning and long-term potentiation in mice. Proc. Natl. Acad. Sci. U.S.A. 96 13427-13431.

van Praag, H., Kempermann, G., and Gage, F.H. (1999b). Running increases cell proliferation and neurogenesis in the adult mouse dentate gyrus. Nat. Neurosci. 2, 266-270.

van Praag, H., Shubert, T., Zhao, C., and Gage, F. H. (2005). Exercise enhances learning and hippocampal neurogenesis in aged mice. J. Neurosci. 25 8680-8685.

Veyrac, A., Sacquet, J., Nguyen, V., Marien, M., Jourdan, F., and Didier, A. (2009). Novelty determines the effects of olfactory enrichment on memory and neurogenesis through noradrenergic mechanisms. Neuropsychopharmacology 34, 786-795.

Wang, S., Scott, B. W., and Wojtowicz, J. M. (2000). Heterogeneous properties of dentate granule neurons in the adult rat. J. Neurobiol. 42, 248-257.

Watters, J. V. (2009). Toward a predictive theory for environmental enrichment. Zoo Biol. 28, 609-622.

Wilson, R.S., Mendes De Leon, C.F., Barnes, L. L., Schneider, J. A., Bienias, J. L., Evans, D. A., and Bennett, D. A. (2002). Participation in cognitively stimulating activities and risk of incident Alzheimer disease. JAMA 287, 742-748.

Wiskott, L., Rasch, M.J., and Kempermann, G. (2006). A functional hypothesis for adult hippocampal neurogenesis: avoidance of catastrophic interference in the dentate gyrus. Hippocampus 16 , 329-343.

Wu, C. W., Chang, Y. T., Yu, L., Chen, H. I., Jen, C. J., Wu, S. Y., Lo, C. P., and Kuo, Y. M. (2008). Exercise enhances the proliferation of neural stem cells and neurite growth and survival of neuronal progenitor cells in dentate gyrus of middle-aged mice. J. Appl. Physiol. 105, 1585-1594.

Conflict of Interest Statement: The authors declare that the research was conducted in the absence of any commercial or financial relationships that could be construed as a potential conflict of interest.

Received: 13 August 2010; paper pending published: 30 September 2010; accepted: 23 October 2010; published online: 08 December 2010.

Citation: Kempermann G, Fabel K, Ehninger D, Babu H, Leal-Galicia P, Garthe $A$ and WolfSA (2010) Why and how physical activity promotes experience-induced brain plasticity. Front. Neurosci. 4:189. doi: 10.3389/fnins.2010.00189

Copyright (c) 2010 Kempermann, Fabel, Ehninger, Babu, Leal-Galicia, Garthe and Wolf. This is an open-access article subject to an exclusive license agreement between the authors and the Frontiers Research Foundation, which permits unrestricted use, distribution, and reproduction in any medium, provided the original authors and source are credited. 